\title{
REBECA trial: a new piece of the evidence for bevacizumab in platinum-resistant recurrent ovarian cancer
}

\author{
Daniele Lavacchi ${ }^{1}$, Sara Fancelli ${ }^{1}$, Marta Rita Gatta Michelet ${ }^{1}$, Martina Catalano ${ }^{1}$, Giandomenico Roviello ${ }^{2}$ \\ ${ }^{1}$ School of Human Health Sciences, ${ }^{2}$ Department of Health Sciences, University of Florence, Florence, Italy \\ Correspondence to: Giandomenico Roviello, MD, PhD. Department of Health Sciences, University of Florence, Viale Pieraccini, 6, 50139, Florence, \\ Italy. Email: giandomenicoroviello@gmail.com. \\ Comment on: Lee JY, Park JY, Park SY, et al. Real-world effectiveness of bevacizumab based on AURELIA in platinum-resistant recurrent ovarian \\ cancer (REBECA): A Korean Gynecologic Oncology Group study (KGOG 3041). Gynecol Oncol 2019;152:61-7.
}

Submitted Nov 07, 2019. Accepted for publication Dec 23, 2019.

doi: $10.21037 /$ tcr.2019.12.83

View this article at: http://dx.doi.org/10.21037/tcr.2019.12.83

Lee et al. (1) evaluated the efficacy and safety of bevacizumab in combination with single-agent chemotherapy for platinum-resistant ovarian cancer in a large retrospective analysis involving Korean women with platinum-resistant recurrent ovarian cancer from 27 different institutions. All evaluated patients had a platinum-resistant recurrent ovarian cancer treated with a single-agent chemotherapy in combination with bevacizumab, independently chose by the investigators, after progression from one or two lines of previous therapies. The chemotherapy included weekly paclitaxel, topotecan, or pegylated liposomal doxorubicin. The primary endpoint was progression free survival (PFS), the secondary endpoints were overall response rate (ORR); PFS2 (calculated as the time from the start of chemotherapy to the occurrence of objective disease progression on nextline therapy or death from any cause), overall survival (OS) and safety/tolerability. From August 2015 to August 2017, a total of 391 women were retrospectively evaluated. Two hundred and fifty-nine (66.2\%) received bevacizumab with pegylated liposomal doxorubicin, 94 (24.0\%) with topotecan, and $38(9.7 \%)$ with weekly paclitaxel. The median duration of treatment was more than one year (13.6 months; range, 1-45 months) for the entire population. In this study, ORR was $32.9 \%$ (31.3\% in second-line therapy and $34.9 \%$ in third-line therapy). In subgroup analysis, the ORR was significantly higher only for the weekly paclitaxel group compared with pegylated liposomal doxorubicin group (48.6\% versus $30.6 \%, \mathrm{P}=0.008)$. The median PFS of the overall population was 6.1 months with the longer value for the weekly paclitaxel cohort. Median OS was 22 months for the entire population with a significant advantage in
OS for the topotecan cohort compared with the pegylated liposomial doxorubicin cohort $(\mathrm{P}=0.018)$. Regarding safety, the rate of adverse events from bevacizumab were in line with those usually reported (2) while the pegylated liposomal doxorubicin cohort had fewer grade $\geq 3$ adverse events however, only the $7.4 \%$ of all patients discontinued treatment because of adverse events from bevacizumabcontaining regimens. The authors concluded that the safety and effectiveness of chemotherapy with bevacizumab in Korean platinum resistant ovarian cancer patients were consistent with previous experiences (1).

To date, the treatment of patients with platinum-resistant ovarian cancer is challenging and often unsuccessful. Several phase 2 trial have explored the efficacy of bevacizumab in combination with chemotherapy, showing promising rates of response and encouraging survival outcomes (3). The only randomized phase 3 study was AURELIAl, which demonstrated a statistically significant benefit in terms of PFS (primary endpoint) and ORR from the addition of bevacizumab to chemotherapy (pegylated liposomal doxorubicin, weekly paclitaxel, or topotecan). PFS and ORR were 6.7 months and $27.3 \%$ in patients treated with bevacizumab plus chemotherapy compared with 3.4 months and $11.8 \%$ in patients treated with chemotherapy alone (PFS HR $0.48, \mathrm{P}<0.001)$. Nevertheless, one of the main factor that has limited the applicability of AURELIA trial was that a statistically significant difference in OS was not achieved. In platinum-resistant patients, usually associated with a poor prognosis and with few therapeutic options, survival should remain one of the most important endpoints for clinical trials, although the OS benefit is regularly underestimated 
due to cross over (40\% in AURELIA trial) (4). However, an exploratory analysis of the AURELIA trial supported the efficacy of bevacizumab in terms of OS in platinum-resistant patients. In this analysis, a survival benefit was observed in patients who received bevacizumab upfront or at crossover compared to those who never received bevacizumab (5).

Targeting tumor angiogenesis, bevacizumab remains an effective option for the treatment of patients with advanced ovarian cancer (2). However, reliable predictive biomarkers for bevacizumab had not yet been identified. Despite recent advances in molecular genome sequencing as a support to therapeutic choices, it remains unclear whether it is better to adopt a molecular or clinical selection of patients. For this purpose, a whole genome gene expression analysis in a subset of patients treated with bevacizumab within ICON7 trial showed a remarkable PFS benefit offered by the use of bevacizumab in patients with proliferative tumors (HR 0.55, $\mathrm{P}=0.016$ ) compared with mesenchymal (HR 0.78, $\mathrm{P}=0.41$ ), immunoreactive (HR 0.67, $\mathrm{P}=0.08$ ), or differentiated tumor (HR 0.85, $\mathrm{P}=0.61)$ (6).

The treatment of ovarian cancer has undergone remarkable changes also with the introduction of poly(ADP-ribose) polymerase (PARP) inhibitors. However, if in BRCA-mutated, platinum-sensitive patients the disease control rate (DCR) was $69-81 \%$, it drops to $39-45 \%$ in platinum-resistant and $23-29 \%$ in platinumrefractory patients $(7,8)$. In addition, several trials have explored combination treatments such as anti-PD-1/PDL1 monoclonal antibodies (MoAbs) with PARP inhibitor, anti-angiogenic agents, or chemotherapy (9-11). In a recent phases 1 and 2 trial, niraparib in combination with pembrolizumab, (anti-PD-1 MoAb) demonstrated a promising activity in patients with recurrent platinumresistant ovarian cancer, with ORR of $18 \%$ and DCR of $65 \%$. Similar rates of response were observed in patients who had previously received bevacizumab or had not, and across subgroups based on BRCA or homologous recombination deficiency (HRD) status (9). The combination of nivolumab (anti-PD-1 MoAb) and bevacizumab was evaluated in a phase 2 trial for patients with relapsed ovarian cancer. The activity of this combination was greater in patients with platinum-sensitive than in platinum-resistant cancer, with ORR of $40.0 \%$ and $16.7 \%$, respectively (10). Moreover, in the randomized phase 3 JAVELIN Ovarian 200 trial, the combination of avelumab (anti-PD-L1 MoAb) and pegylated liposomal doxorubicin demonstrated a modest activity in patients with platinum-resistant or refractory ovarian cancer, with ORR of $13.3 \%$ (11).

In conclusion, REBECA trial evaluated the efficacy and safety of bevacizumab with single-agent chemotherapy chosen by the investigators in Korean patients with platinum-resistant ovarian cancer. Several limitations deserve specific attention, mainly due to the intrinsic nature of an observational study. The lack of a control group, the choice of the chemotherapeutic agent based on the preferences of the investigators, the lack of stratification and exploratory analysis of predictive biomarkers represented the major limitations that profoundly affect the strength of the conclusions. In addition, the participation of exclusively Korean centers precludes a comprehensive evaluation of efficacy and tolerability in both Western and Eastern patients. However, this study offers an additional evidence on the use of bevacizumab in routine clinical practice for this subset of patients with ovarian cancer who have few treatment options.

\section{Acknowledgments}

Funding: None.

\section{Footnote}

Provenance and Peer Review: This article was commissioned by the editorial office, Translational Cancer Research. The article did not undergo external peer review.

Conflicts of Interest: All authors have completed the ICMJE uniform disclosure form (available at http://dx.doi. org/10.21037/tcr.2019.12.83). The authors have no conflicts of interest to declare.

Ethical Statement: The authors are accountable for all aspects of the work in ensuring that questions related to the accuracy or integrity of any part of the work are appropriately investigated and resolved.

Open Access Statement: This is an Open Access article distributed in accordance with the Creative Commons Attribution-NonCommercial-NoDerivs 4.0 International License (CC BY-NC-ND 4.0), which permits the noncommercial replication and distribution of the article with the strict proviso that no changes or edits are made and the original work is properly cited (including links to both the formal publication through the relevant DOI and the license). See: https://creativecommons.org/licenses/by-nc-nd/4.0/. 


\section{References}

1. Lee JY, Park JY, Park SY, et al. Real-world effectiveness of bevacizumab based on AURELIA in platinumresistant recurrent ovarian cancer (REBECA): A Korean Gynecologic Oncology Group study (KGOG 3041). Gynecol Oncol 2019;152:61-7.

2. Roviello G, Bachelot T, Hudis CA, et al. The role of bevacizumab in solid tumours: A literature based metaanalysis of randomised trials. Eur J Cancer 2017;75:245-58.

3. McClung EC, Wenham RM. Profile of bevacizumab in the treatment of platinum-resistant ovarian cancer: current perspectives. Int J Womens Health 2016;8:59-75.

4. Pujade-Lauraine E, Hilpert F, Weber B, et al. Bevacizumab combined with chemotherapy for platinum-resistant recurrent ovarian cancer: The AURELIA open-label randomized phase III trial. J Clin Oncol 2014;32:1302-8.

5. Bamias A, Gibbs E, Khoon Lee C, et al. Bevacizumab with or after chemotherapy for platinum-resistant recurrent ovarian cancer: exploratory analyses of the AURELIA trial. Ann Oncol 2017;28:1842-8.

6. Kommoss S, Winterhoff B, Oberg AL, et al. Bevacizumab May Differentially Improve Ovarian Cancer Outcome in Patients with Proliferative and Mesenchymal Molecular Subtypes. Clin Cancer Res 2017;23:3794-801.

7. Fong PC, Yap TA, Boss DS, et al. Poly(ADP)-ribose

Cite this article as: Lavacchi D, Fancelli S, Gatta Michelet MR, Catalano M, Roviello G. REBECA trial: a new piece of the evidence for bevacizumab in platinum-resistant recurrent ovarian cancer. Transl Cancer Res 2020;9(2):402-404. doi: 10.21037/tcr.2019.12.83 polymerase inhibition: frequent durable responses in BRCA carrier ovarian cancer correlating with platinumfree interval. J Clin Oncol 2010;28:2512-9.

8. Konecny GE, Ozo AM, Tinker AV, et al. Rucaparib in patients with relapsed, primary platinum-sensitive highgrade ovarian carcinoma with germline or somatic BRCA mutations: integrated summary of efficacy and safety from the phase 2 study ARIEL2 (NCT01891344). Gynecol Oncol 2017;145:2.

9. Konstantinopoulos PA, Waggoner S, Vidal GA, et al. Single-Arm Phases 1 and 2 Trial of Niraparib in Combination With Pembrolizumab in Patients With Recurrent Platinum-Resistant Ovarian Carcinoma. JAMA Oncol 2019. [Epub ahead of print].

10. Liu JF, Herold C, Gray KP, et al. Assessment of Combined Nivolumab and Bevacizumab in Relapsed Ovarian Cancer: A Phase 2 Clinical Trial. JAMA Oncol 2019. [Epub ahead of print].

11. Pujade-Lauraine E, Fujiwarab K, Ledermann JA, et al. Avelumab alone or in combination with pegylated liposomal doxorubicin versus pegylated liposomal doxorubicin alone in platinum-resistant or refractory epithelial ovarian cancer: Primary and biomarker analysis of the phase III JAVELIN Ovarian 200 trial. Gynecol Oncol 2019;154:21-2. 